\title{
Sound Radiation from a Generic Bypass Duct with Bifurcations
}

\author{
Xiaoxian Chen ${ }^{1}$, Xun Huang ${ }^{2}$ and Xin Zhang ${ }^{3}$ \\ School of Engineering Sciences, \\ Southampton University, \\ Southampton, SO17 1BJ, United Kingdom
}

\begin{abstract}
The influence of bifurcations in an aero-engine bypass duct on noise radiation was investigated through high-order accurate three-dimensional numerical simulations. The physical process was described by a set of acoustic perturbation equations (APE), with a background mean flow. Four bifurcation arrangements, with an airfoil cross-section, were regularly placed in circumferential direction. Results of the simulations were compared with those of a clean duct case. A circumferential mode of $m=12$ with radial mode of $n=1$ and multi- $n$ modes at a source frequency of $1547 \mathrm{~Hz}$ were set as acoustic inputs. For single radial mode case, acoustic modes interfered with the bifurcations resulting in a doubled circumferential mode pattern in the near field acoustic pressure distribution. Far field noise computations indicated $3.2 \mathrm{~dB}$ and $2.0 \mathrm{~dB}$ sound pressure level increases for radial mode of $n=1$ and multi- $n$ modes respectively. A comparison was also made between APE and linearlised Euler equations (LEE). Results showed that the APE simulation produced almost identical far field sound prediction to the LEE, with a small difference $(<0.8 \mathrm{~dB})$ at the main radiation peak angle.
\end{abstract}

\section{Introduction}

Engine noise is an important component of aircraft noise, along with high-lift and landing gear noise. For engines with high bypass ratio, fan noise is a major noise source. Accurate simulation of noise propagation within the engine duct and estimation of far field noise radiation are necessary to provide useful numerical tools for development of noise attenuation methods. A significant amount of research has been undertaken to develop computational methods for engine noise problem using either frequency domain ${ }^{1}$ or time domain method ${ }^{2-3}$. For cases with an axisymmetric mean flow environment, a two-and-half dimensional (2.5D) linearsied Euler equations (LEE) model $^{2}$ was used in previous works to solve three-dimensional (3D) spinning mode duct radiation problems on a two-dimensional (2D) domain. The model was validated by comparing its solution to an analytical solution in a straight duct case. To avoid numerical instability excited in the mean flow shear layer aft duct exit, a revised LEE model was presented

\footnotetext{
${ }^{1}$ Research Fellow.

${ }^{2}$ Lecturer, AIAA member.

${ }^{3}$ Professor, Associated Fellow, AIAA.
} 
by removing a couple of terms in the LEE model ${ }^{2}$. However, the physical meaning of the modification is unclear. Through applying an acoustic filtering on the LEE model, a new model with a set of so called acoustic perturbation equations (APE) ${ }^{4}$ have been developed in recent years. The APE model removes vortical modes and thus suppresses numerical instabilities in the sheared flow. A 2.5D APE model was used in the literature ${ }^{3}$ for duct cases with an axisymmetric mean flow environment.

In this work, the capacity of the current aeroacoustic code was extended to cater for more realistic geometries and flow/acoustic conditions, such as scarf nozzles. A 3D APE model was implemented for both Cartesian and cylindrical coordinate systems. The 3D model was validated by comparing its results with those of the 2.5D APE model in an axisymmetric case.

Another part of the work addressed the effect of bifurcations. Devices such as bifurcations, installed in the engine bypass duct exert influence on the spinning mode propagation pattern and mode strength ${ }^{1,5-6}$. Nark et al. ${ }^{5}$ studied bifurcations, with a cross-section of NACA0012 airfoil shape, in a generic engine bypass duct using a CDUCT-LaRC code which was based on a parabolic approximation to the convected Helmholtz equation. A non-uniform background mean flow was obtained with Mach number 0.4 at the duct inlet. The radiation predictions were predicted at $1.9 \mathrm{~m}$ away from the origin of the duct exit. A case of four combined spinning modes, $(m=5, n=1),(m=5, n=2),(m=10, n=1)$ and $(m=10, n=2)$, with a source frequency of $3000 \mathrm{~Hz}$ was studied, where $m$ is the circumferential mode and $n$ the radial mode. They found that the bifurcations did affect the modal content in both the propagation and radiation results. They also found that liner treatment on the bifurcation clearly reduced the radiation angle so that the radiation was mainly along the downstream direction. In another study of bifurcation effect, Baralon et al. ${ }^{1}$ used a 2D meridional LEE code which was based on Fourier decomposition in the circumferential direction on frequency domain and was applied to a limited number of 2D meridional planes to save computing time. A flat plate bifurcation was placed in a straight annual duct. A uniform axial mean flow of Mach number 0.44 was utilized in the study. In a multi- $m$ mode case, from mode $m=-22$ to 22 , they reported that mode energy transfer from $m=4$ mode to higher or lower modes which was consistent with the conclusions of Nark et al. ${ }^{5}$. Sugimoto et $a .^{6}$ also conducted a numerical simulation using a finite element method to study the bifurcation effect. Four modes, $(m=0, n=1),(m=9, n=1),(m=15, n=1)$ and $(m=18, n=1)$, with source frequencies from $500 \mathrm{~Hz}$ to $1000 \mathrm{~Hz}$, were studied. Two bifurcations (roughly a rectangular shape) were regularly placed in the bypass duct. Their study on thickness of the bifurcation revealed that the thicker of the bifurcation the more energy was reflected. The liner treatment gave a maximum $2 \mathrm{~dB}$ reduction in sound power level.

In all the three works listed above efforts were placed either on demonstrating the robustness of computation codes or on liner effect. There was no comparison of bifurcation effect in near field or far field in terms of acoustic radiation pattern and strength change. In this work the 3D APE code is applied to a case with four bifurcations fitted in a generic engine bypass duct to study the bifurcation effect in terms of near field sound pressure level (SPL) and far field radiation directivity. 


\section{Numerical Methods and Model Setup}

\section{A. APE and LEE formulations}

The 2.5D $\mathrm{APE}^{3}$ can be presented as,

$$
\begin{aligned}
& \frac{\partial \rho^{\prime}}{\partial t}+\frac{\partial\left(\rho^{\prime} u_{0}+\rho_{0} u^{\prime}\right)}{\partial x}+\frac{\partial\left(\rho^{\prime} v_{0}+\rho_{0} v^{\prime}\right)}{\partial r}-\frac{m \rho_{0}}{k r} w_{t}^{\prime}+\frac{\rho^{\prime} v_{0}+\rho_{0} v^{\prime}}{r}=0, \\
& \frac{\partial u^{\prime}}{\partial t}+\frac{\partial}{\partial x}\left(u_{0} u^{\prime}+v_{0} v^{\prime}+p^{\prime} / \rho_{0}\right)=q_{m, x}=\left[\left(\frac{\partial v_{0}}{\partial x}-\frac{\partial u_{0}}{\partial r}\right) v^{\prime}+\left(\frac{\partial v^{\prime}}{\partial x}-\frac{\partial u^{\prime}}{\partial r}\right) v_{0}\right], \\
& \frac{\partial v^{\prime}}{\partial t}+\frac{\partial}{\partial r}\left(u_{0} u^{\prime}+v_{0} v^{\prime}+p^{\prime} / \rho_{0}\right)=q_{m, r}=-\left[\left(\frac{\partial v_{0}}{\partial x}-\frac{\partial u_{0}}{\partial r}\right) u^{\prime}+\left(\frac{\partial v^{\prime}}{\partial x}-\frac{\partial u^{\prime}}{\partial r}\right) u_{0}\right], \\
& \frac{\partial w_{t}^{\prime}}{\partial t}+u_{0} \frac{\partial w_{t}^{\prime}}{\partial x}+v_{0} \frac{\partial w_{t}^{\prime}}{\partial r}+\frac{m k}{\rho_{0} r} p^{\prime}+\frac{w_{t}^{\prime} v_{0}}{r}=0, \quad\left(\text { with } \quad w_{t}^{\prime}=\frac{\partial w^{\prime}}{\partial t}\right),
\end{aligned}
$$

where superscript $(')$ and subscript $(0)$ denote perturbation and mean properties respectively; $u$, $v^{\prime}$ and $w^{\prime}$ are velocity perturbations in the axial $(x)$, radial $(r)$ and circumferential $(\theta)$ directions respectively. The fluid is modeled as a perfect gas and homentropic assumption applies, i.e. $p^{\prime}=C_{0}^{\prime} \rho^{\prime}$. In Eq. (1) an axisymmetric background mean flow is assumed, i.e. $w_{0}=0$. Terms $q_{m, x}$ and $q_{m, r}$ are source terms due to existence of a shear flow. The main objective of this work was to study the spinning mode propagation in the near field, refractions in the sheared flow, and radiation to the far field. Mean flow sound sources were not considered and set to zero in the current computation.

To compare with the 2.5D APE shown above, the revised $2.5 \mathrm{D} \mathrm{LEE}^{2}$ is also presented in the cylindrical coordinates as

$$
\begin{aligned}
& \frac{\partial \rho^{\prime}}{\partial t}+\frac{\partial\left(\rho^{\prime} u_{0}+\rho_{0} u^{\prime}\right)}{\partial x}+\frac{\partial\left(\rho^{\prime} v_{0}+\rho_{0} v^{\prime}\right)}{\partial r}-\frac{m \rho_{0}}{k r} w_{t}^{\prime}+\frac{\rho^{\prime} v_{0}+\rho_{0} v^{\prime}}{r}=0, \\
& \frac{\partial u^{\prime}}{\partial t}+u_{0} \frac{\partial u^{\prime}}{\partial x}+v_{0} \frac{\partial u^{\prime}}{\partial r}+\left(u^{\prime}+\frac{\rho^{\prime}}{\rho_{0}} u_{0}\right) \frac{\partial u_{0}}{\partial x}+\frac{\partial p^{\prime}}{\rho_{0} \partial x}=0, \\
& \frac{\partial v^{\prime}}{\partial t}+u_{0} \frac{\partial v^{\prime}}{\partial x}+v_{0} \frac{\partial v^{\prime}}{\partial r}+\left(u^{\prime}+\frac{\rho^{\prime}}{\rho_{0}} u_{0}\right) \frac{\partial v_{0}}{\partial x}+\left(v^{\prime}+\frac{\rho^{\prime}}{\rho_{0}} v_{0}\right) \frac{\partial v_{0}}{\partial r}+\frac{\partial p^{\prime}}{\rho_{0} \partial r}=0, \\
& \frac{\partial w_{t}^{\prime}}{\partial t}+u_{0} \frac{\partial w_{t}^{\prime}}{\partial x}+v_{0} \frac{\partial w_{t}^{\prime}}{\partial r}+\frac{m k}{\rho_{0} r} p^{\prime}+\frac{w_{t}^{\prime} v_{0}}{r}=0 .
\end{aligned}
$$

A detailed derivation of the 2.5D LEE and the 2.5D APE can be found in Zhang et al. ${ }^{2}$ and Huang et $a l^{3}$. The robustness and accuracy of this treatment were demonstrated through a comparison with analytical solutions using benchmark test cases ${ }^{2}$. For generic test cases, results of a LEE simulation could be different from those of the APE with a background mean flow. In the late validation section, a comparison is made between the 2.5D APE and the 2.5D LEE. 
For non- axisymmetrical cases, the 3D APE model was used. It is as follows:

$$
\begin{aligned}
& \frac{\partial \rho^{\prime}}{\partial t}+\frac{\partial\left(\rho^{\prime} u_{0}+\rho_{0} u^{\prime}\right)}{\partial x}+\frac{\partial\left(\rho^{\prime} v_{0}+\rho_{0} v^{\prime}\right)}{\partial r}+\frac{\partial\left(\rho^{\prime} w_{0}+\rho_{0} w^{\prime}\right)}{r \partial \theta}+\frac{\rho_{0} v^{\prime}+\rho^{\prime} v_{0}}{r}=0, \\
& \frac{\partial u^{\prime}}{\partial t}+\frac{\partial}{\partial x}\left(u^{\prime} u_{0}+v_{0} v^{\prime}+w_{0} w^{\prime}+\frac{p^{\prime}}{\rho_{0}}\right)=q_{m, x}, \\
& \frac{\partial v^{\prime}}{\partial t}+\frac{\partial}{\partial r}\left(u^{\prime} u_{0}+v_{0} v^{\prime}+w_{0} w^{\prime}+\frac{p^{\prime}}{\rho_{0}}\right)-\frac{2 w_{0} w^{\prime}}{r}=q_{m, r}, \\
& \frac{\partial w^{\prime}}{\partial t}+\frac{\partial}{r \partial \theta}\left(u^{\prime} u_{0}+v_{0} v^{\prime}+w_{0} w^{\prime}+\frac{p^{\prime}}{\rho_{0}}\right)+\frac{v_{0} w^{\prime}+v^{\prime} w_{0}}{r}=q_{m, \theta} .
\end{aligned}
$$

For the same reason addressed in the 2.5D APE, source terms $\left(q_{m, x}, q_{m, r}\right.$ and $\left.q_{m, \theta}\right)$ are omitted in this work.

\section{B. Numerical Schemes and Model Setup}

In the computations, the temporal scheme used is a low-dissipation and dispersion Runge-Kutta scheme $^{7}$ and the spatial scheme is a $6^{\text {th }}$-order accurate compact scheme ${ }^{8}$. An explicit filter of $10^{\text {th }}$-order accuracy ${ }^{9}$ is applied at every time step to filter out small numerical disturbances. Inflow and outflow buffer-zones ${ }^{2}$ are placed in the inflow and outflow areas to minimize possible spurious wave reflections at the computational boundaries. The inflow buffer-zone also acts as a spinning mode input region with the boundary conditions, which can be found in literatures ${ }^{2-3}$. A periodic boundary condition is applied at the boundaries in the circumferential direction except the bifurcation section. A slip-wall condition is applied to all wall sections including the bifurcation section. For computations in a cylindrical coordinate system a symmetric flow condition is applied at the axis. A circumferential mode of $m=12$ was chosen so that an acoustic grid consisting of a quarter-circumferential plane, containing three circumferential modes, can be established for a bifurcation case. In order to assess the effect of bifurcations, a clean bypass duct case was also computed using the 2.5D APE and the LEE respectively. Non-dimensional acoustic frequency was set to 28 (that is $1547 \mathrm{~Hz}$ ). Two radial mode cases were studied. The first case had a radial mode of $n=1$ and the second had multi- $n$ mode consisting of five cut-on downstream propagating radial modes from $n=1$ to 5 . Estimation of far field acoustic radiation was performed separately based upon the near field computation results through an integral formula of Ffowcs Williams-Hawkings (FW-H) equation ${ }^{10}$. The far field directivity was calculated at $100 \mathrm{~m}$ away from the bypass duct exit.

A quarter of the aft engine duct configuration is illustrated in Figure 1. The geometry includes bifurcations, the bypass and exhaust ducts. Four bifurcations are placed along the circumferential direction at regular angle intervals of $90 \mathrm{deg}$ and the bifurcations are connected with the inner and the outer bypass duct walls in the radial direction. At the duct inlet, the inner wall radius of the exhaust duct is $0.57 \mathrm{~m}$, the exhaust cone radius is $0.23 \mathrm{~m}$, the inner wall of the bypass duct radius is $0.79 \mathrm{~m}$ and the inner hub radius is $0.6 \mathrm{~m}$. In Figure 1 the length of the bypass duct (from the spinning mode entry area to the duct exit) is $4 \mathrm{~m}$. The bifurcations have a cross section of NACA0012 airfoil shape with a chord of $1 \mathrm{~m}$. Starting position of the bifurcations is $3 \mathrm{~m}$ away 
from the bypass duct exit. The installation positions can be adjusted later easily for specific applications.

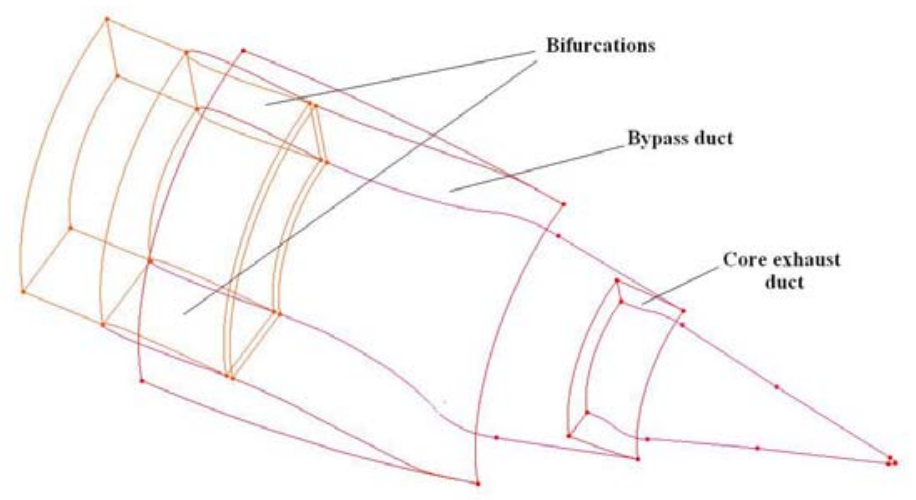

Figure1: A schematic of aft duct with bifurcations.

The computations are performed on structured grids and non-dimensional forms of the equations are employed. For the bifurcation case shown in Figure 2, the acoustic grid is constructed in a quarter-circumferential volume (circumferential angle $\theta$ between 0 and $90 \mathrm{deg}$ ) consisting of $1.27 \times 10^{6}$ cells and the bifurcations are placed at positions of 0 and $90 \mathrm{deg}$. The computational domain has lengths of $7 \mathrm{~m}$ and $20 \mathrm{~m}$ in the radial and the axial directions respectively in a cylindrical coordinate system. The grid resolution has a minimum 9 point-per-wave-length (PPW) in both directions. In the circumferential direction, a 10 PPW grid resolution was used for the $n=1$ and multi- $n$ mode cases as it was found to be necessary to retain accuracy for the multimode case. The axis point is taken away due to restriction on the structured grid and minimum radius is set at $0.05 \mathrm{~m}$. The computation was performed on a Linux PC cluster using 12 processors with $3.06 \mathrm{GHz}$ clock speed each and $14 \mathrm{~GB}$ of memory. A computation of 50 acoustic mode periods required 40 hour computing time for the bifurcation case. In the clean duct case the grid is constructed on a single $m$ mode plane ( $\theta$ between 0 and $30 \mathrm{deg}$ ) consisting of $0.49 \times 10^{6}$ cells, as shown in Figure 3. The simulation was performed on 12 processors for 13 hours. The 2.5D LEE model and the 2.5D APE model were used to solve the clean duct case.

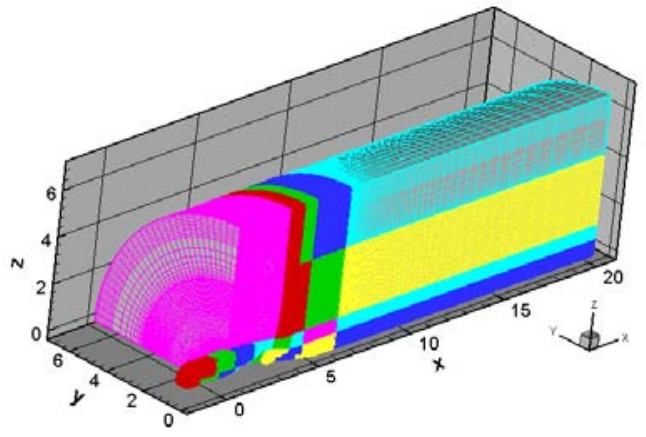

Figure 2: Grid for the bifurcation computation.

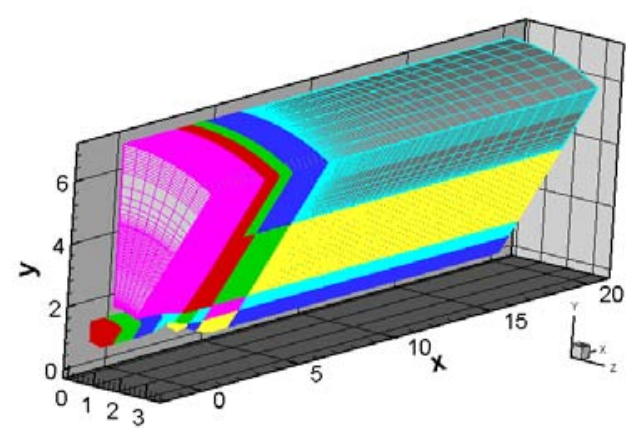

Figure 3: Grid for the clean duct computation. 


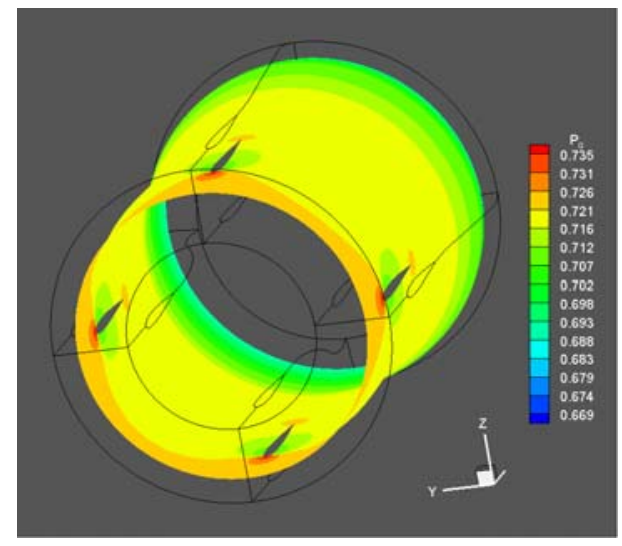

Figure 4: Mean pressure around bifurcations.

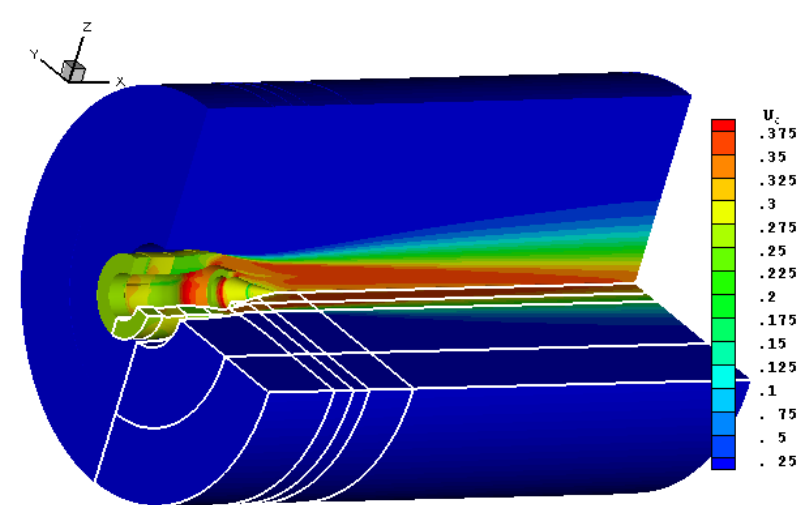

Figure 5: Mean axial velocity distribution.

In high-order acoustic computations, steady background mean flow data were used. The fluid was modeled as a perfect gas. The background mean flow was determined from solutions of the Reynolds Averaged Navier-Stokes with $k-\omega$ turbulence model. Two background mean flows were simulated for a clean bypass duct case (without bifurcations) and a bifurcation case. The clean duct case was used to validate the 3D APE implementation and also to compare with the bifurcation case. For both cases inflow conditions were: at the bypass duct inlet a pressure of $112058 \mathrm{~Pa}$ and a Mach number of 0.27 ; at freestream a pressure of $101325 \mathrm{~Pa}$ and no flow. Temperature was a constant of $299.2 \mathrm{~K}$. Shown in Figures 4 and 5 are the mean pressure and the mean axial velocity contours for the bifurcation case. These mean flow data were interpolated onto the grids for acoustic computation. Since the presence of the boundary layer was not considered in the acoustic computation, the grids were not refined near the duct walls. The mean flow velocity values were the same in the radial direction at first three grid points near the duct walls. In acoustic computations all variables were nondimensionalised using a reference length of $1 \mathrm{~m}$, a reference density $1.225 \mathrm{~kg} / \mathrm{m}^{3}$, and a reference sound speed of $346.76 \mathrm{~m} / \mathrm{s}$.

\section{Validation of 3D APE model}

Validation of the 3D APE code was conducted through a comparison with results of a 2.5D $\mathrm{APE}^{3}$ computation in a clean duct case (without bifurcations) for the $n=1$ mode. Figure 6 shows SPL contours which were calculated in every grid point with a reference pressure of $2 \times 10^{-5} \mathrm{~Pa}$. It can be seen from Figure 6 that the near field SPL from the 3D APE simulation is consistent with the result of the 2.5D APE in terms of the SPL pattern. Inside the duct the maximum SPL value of $103.7 \mathrm{~dB}$ is less than that of the 2.5D APE $(104.5 \mathrm{~dB})$. Far field directivity is predicted at $100 \mathrm{~m}$ away from the bypass duct exit shown in Figure 7. A zenith $(\varphi)$ angle is used to represent radiation angle from the duct exit. It is evident that the 3D APE results match the 2.5D APE results with slightly angle phase shifts within main radiation peak range. Two major radiation peaks are observed for the 3D APE and they are $61.5 \mathrm{~dB}$ at $40.0 \mathrm{deg}$ and $60.6 \mathrm{~dB}$ at $44.9 \mathrm{deg}$. Compared with two mean peak values for the 2.5D APE, $60.5 \mathrm{~dB}$ at $41.2 \mathrm{deg}$ and 61.0 $\mathrm{dB}$ at $46.0 \mathrm{deg}$, the maximum differences are $0.5 \mathrm{~dB}$ in strength and $-1.2 \mathrm{deg}$ in phase. At the first interference dip the SPL values are $34.7 \mathrm{~dB}$ at $48.5 \mathrm{deg}$ for the 3D APE and $35.5 \mathrm{~dB}$ at 49.7 deg for the 2.5D APE. The differences are $-0.8 \mathrm{~dB}$ and -1.2 deg respectively. For this validation only difference between the $3 \mathrm{D}$ APE and the $2.5 \mathrm{D}$ APE is the circumferential grid resolution. 
Theoretically an infinite circumferential grid resolution is assumed for the 2.5D form of the equations but in reality a certain grid resolution is applied when far field directivity is calculated. In this validation a $20 \mathrm{PPW}$ grid resolution is used to construct a 3D integration surface while for the 3D APE the grid resolution is $10 \mathrm{PPW}$. Less grid resolution in the 3D APE results in dispersion. However it can be conclude that the 3D APE implementation is correct since the results are close to the 2.5D APE results either in the near field or the far field.

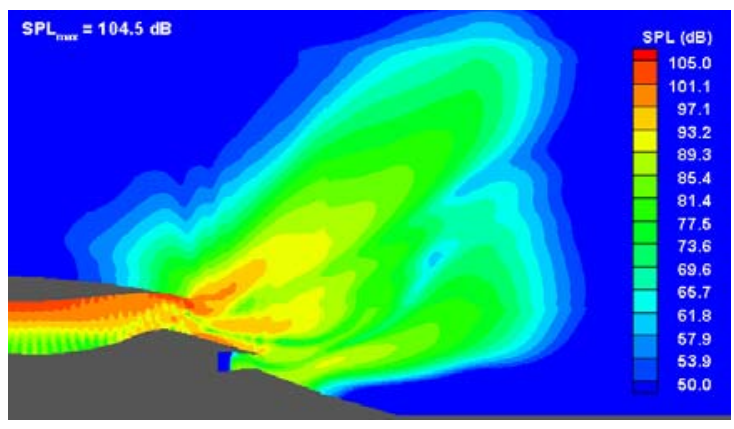

(a): 2.5D APE

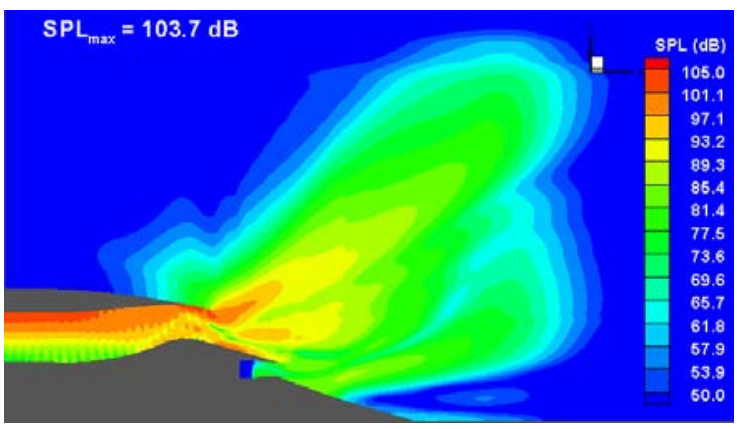

(b): 3D APE.

Figure 6: SPL contours in a clean duct.

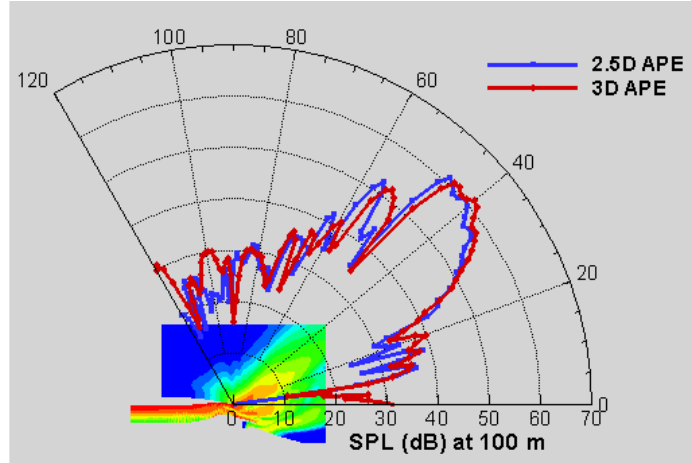

Figure 7: 3D APE and 2.5D APE computation of $(m=12, n=1)$ mode: far field directivities.

It is also of interest to see a comparison between the 2.5D APE and the 2.5D LEE predictions (Figure 8). The results of the both computations agree quite well in terms of the near field SPL pattern. However the 2.5D APE computation produces a higher SPL value $(0.2 \mathrm{~dB})$ over the LEE in comparison to the maximum SPL value (104.3 dB for the LEE) inside the duct. In this work the LEE produces a slightly lower sound pressure level not only in near field but also in far field, as seen in the far field directivity (Figure 9). Overall, however, the two sets of equations produce similar predictions. 


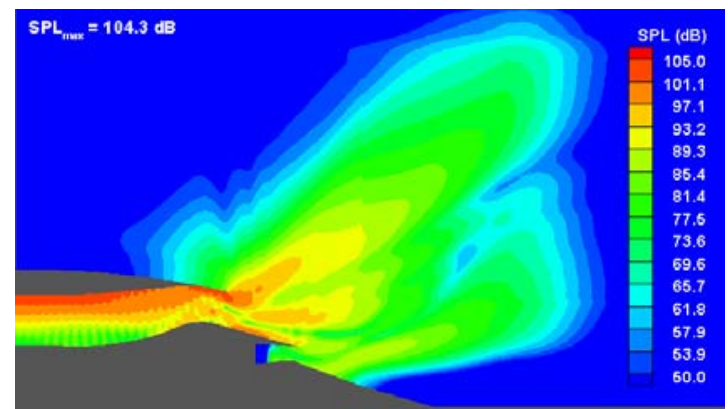

Figure 8: SPL contours in a clean duct. (2.5D LEE)

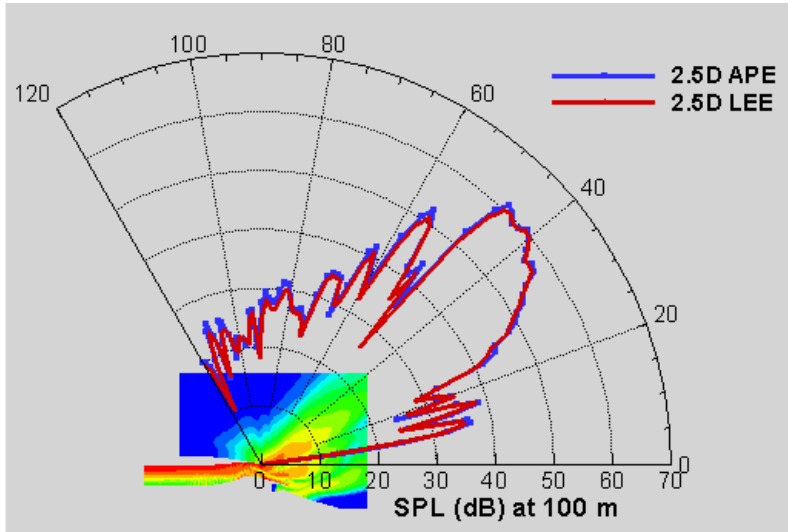

Figure 9: 2.5D computation of $(m=12, n=1)$ mode: far field directivity comparison. 


\section{Results of Bifurcation Cases}

A. $n=1$ mode case

Figures 10 to 14 show the near field acoustic pressures, SPL and the far field directivities. In general, the presence of the bifurcations does have significant effect on the acoustic mode propagation path and local strength.

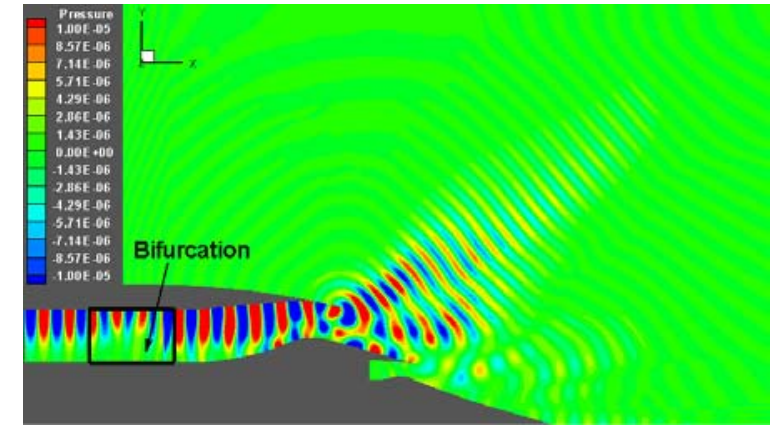

(a) $\theta=0 \mathrm{deg}$

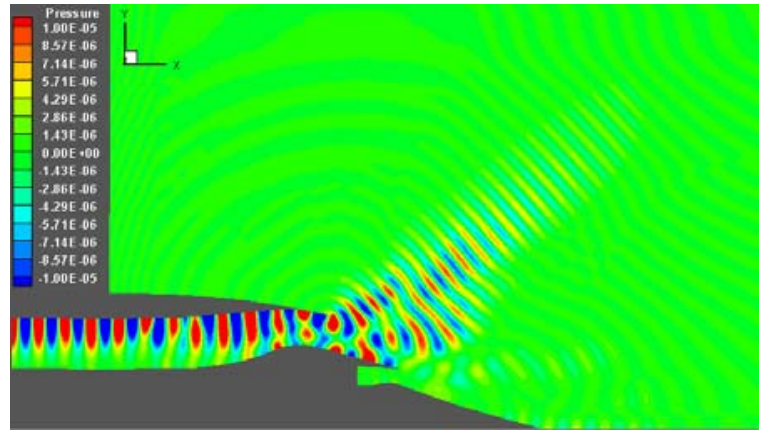

(b) $\theta=45 \operatorname{deg}$

Figure 10 : Acoustic pressure contours.

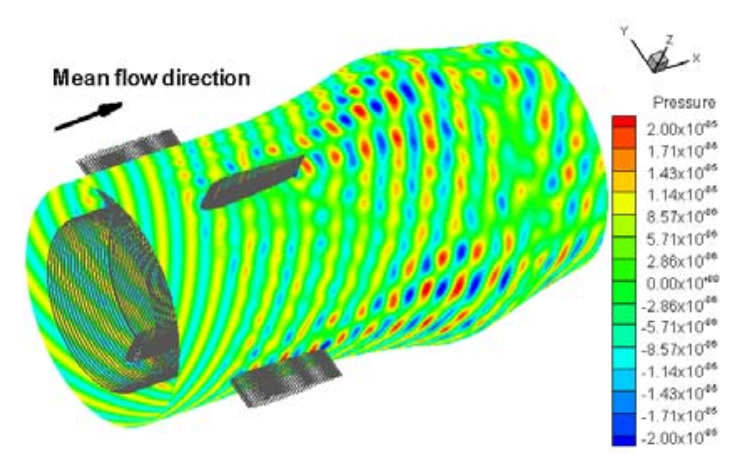

(a) circumferential view

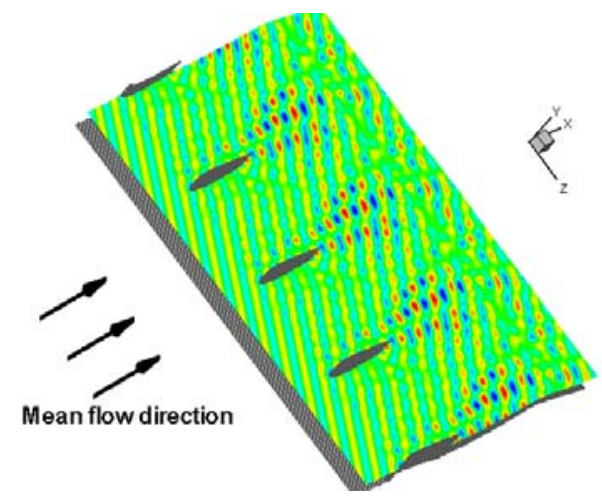

(b) plane ( $x-\theta$ plane) view

Figure 11: A view of acoustic pressures at a cross-section of the bypass duct.

In Figure 10 , a comparison is made between a middle plane ( $\theta=45 \mathrm{deg})$ and a bifurcation plane $(\theta=0 \mathrm{deg})$ along the circumferential direction. In the rear (right) side of the bifurcations the acoustic pressures are stronger and main radiation peaks are also stronger. It is noticed that main radiation angle is not altered as radiation peak angles are almost the same for both $\theta$-planes. Figure 11(b) shows a slice for the acoustic pressure distribution in an $x-\theta$ plane, a circumferentially clockwise moving mode is diffracted from the bifurcations, which results in stronger acoustic pressures when the diffracted modes are interfered with each other behind the bifurcations. From Figure 12 stronger acoustic pressure radiation pattern outside the duct can also be observed at $\theta=0 \mathrm{deg}$. On the SPL strength variation the maximum SPL values inside the duct are $110.5 \mathrm{~dB}$ and $104.3 \mathrm{~dB}$ for the bifurcation case and the clean duct case respectively, giving a $6.2 \mathrm{~dB}$ SPL increase. It is also observed from Figure 13 that a doubled circumferential mode $(m=24)$ SPL pattern is formed at a fixed axial position $(x / L=3.5)$ outside the duct and the 
acoustic pressure contours show that main structure of the $m=12$ mode remains. The doubled $m$ mode SPL pattern is a result of mode interference since without bifurcations the SPL distribution is uniform along the circumferential direction. In the work of Nark et al. ${ }^{5}$ stronger acoustic pressures were indeed observed in the vicinity of the bifurcations. In the work of Baralon et al. ${ }^{6}$ the acoustic pressure pattern was found to be slightly distorted. Both works have shown the presence of stronger acoustic pressure pattern as a result of interference.

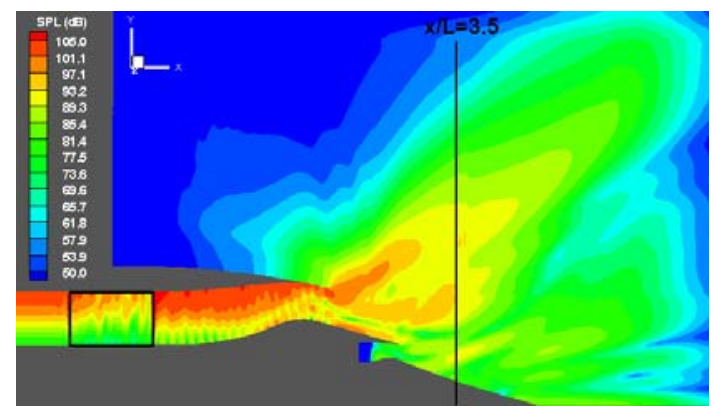

(a) at $\theta=0 \mathrm{deg}$

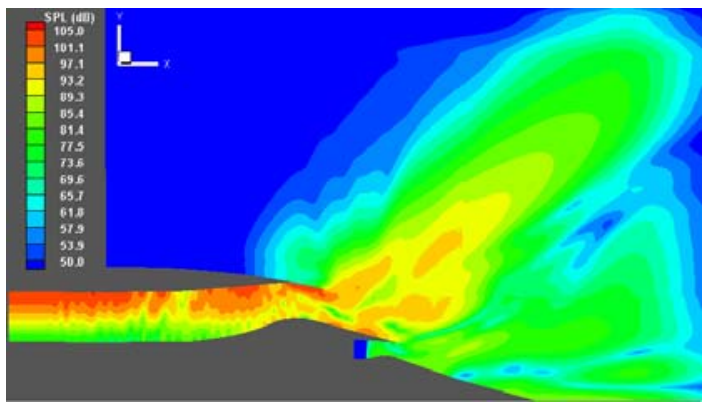

(b) at $\theta=45 \mathrm{deg}$

Figure 12: Near field SPL pattern.

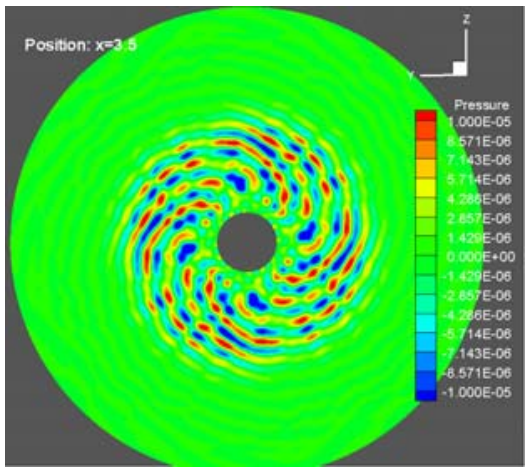

(a) Acoustic pressure

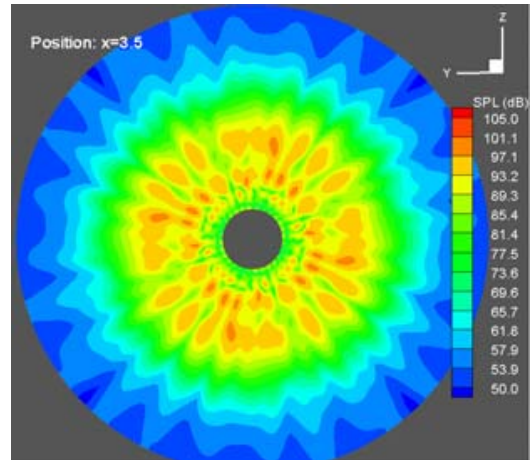

(b) SPL

Figure 13: Acoustic pattern outside the bypass duct at $x / L=3.5$ (position illustrated in Figure 12 (a)). View angle is from positive axial direction (looking right from the duct).

A 3D far field directivity pattern at $100 \mathrm{~m}$ is shown in Figure 14 and the figure displayed is the outward face of the hemisphere centered at a zero zenith angle $(\varphi=0)$. A total number of 10860 observer positions are calculated with a resolution of $2 \mathrm{deg}$ in both the circumferential (0 $<\theta \leqq 360 \mathrm{deg})$ and zenith $(0<\varphi \leqq 120 \mathrm{deg})$ directions in a spherical coordinate system. As only a quarter of the total volume was used in the computation, the individual far field profiles are compared at two cross-sections $(\theta=0$ and $\theta=45 \mathrm{deg})$, which are shown in Figure 15. A nearly doubled $m$ mode SPL pattern appears (Figure 14) in the far field, where about 24 maximum SPL values are counted, showing the bifurcation effect. As shown in Figure 15, the peak radiation angles remain the same and their range is from $\varphi=33$ to $\varphi=48 \mathrm{deg}$. Two maximum SPL values of 63.0 and $62.5 \mathrm{~dB}$ are observed for the $\theta=0$ deg cross-section at radiation angles of $\varphi=41.2$ and $44.9 \mathrm{deg}$ respectively. One maximum SPL value of $63.8 \mathrm{~dB}$ at a radiation angle of $\varphi=41.6$ is observed for the $\theta=45$ deg cross-section. For the clean duct case, two maximum SPL values of 
60.3 and $60.6 \mathrm{~dB}$ are observed for the $\theta=0 \mathrm{deg}$ cross-section at radiation angles of $\varphi=41.2$ and $44.8 \mathrm{deg}$ respectively. The maximum SPL increase is $3.2 \mathrm{~dB}$ in comparison to the clean duct case. In addition, the bifurcation case has higher sound pressure levels in lower radiation angles ( $\varphi=0$ and $\varphi=20 \mathrm{deg}$ ).

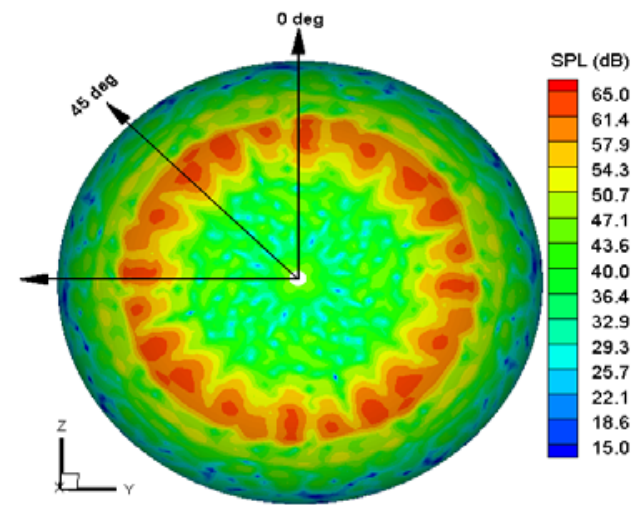

Figure 14: Directivity at far field. 0 and $45 \mathrm{deg}$ indicate circumferential angles.

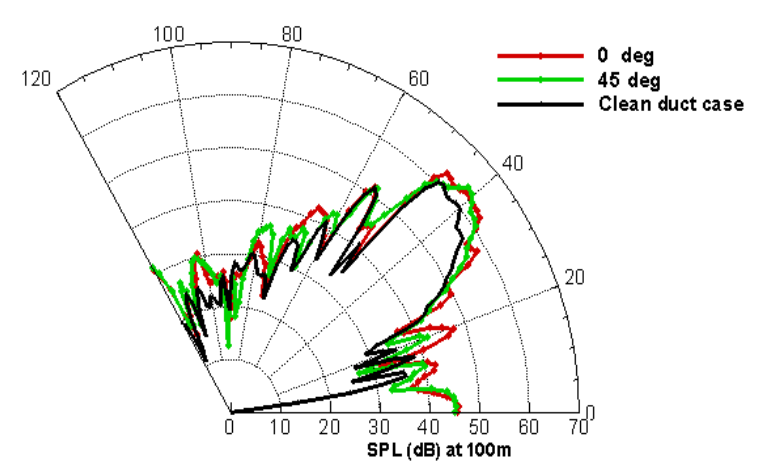

Figure 15: Directivities in the zenith $(\varphi)$ direction.

\section{B. Multi- $n$ mode case}

For the multi- $n$ mode case, the maximum acoustic pressure amplitude is produced by the $n=3$ radial mode and its position is at the inner duct wall. For the $n=1$ mode, the maximum pressure appears at the duct outer wall. It can be seen from Figure 12 that the duct curvature does not affect the $n=1$ mode propagation significantly except near the vicinity of the bypass duct edge. For the multi- $n$ mode case, complex acoustic propagation patterns and mode interference are expected. In observing the acoustic pressure distribution at the middle plane $(\theta=45 \mathrm{deg})$ and the bifurcation plane $(\theta=0 \mathrm{deg})$ shown in Figure 16, it is noticed that the stronger acoustic pressures appear behind the bifurcations while they appear over the external surface of the core nozzle in the middle plane. Behind the bifurcations strong acoustic modes move towards the outer wall and are then reflected from the edge of the bypass duct resulting in a stronger lower radiation pattern in comparison to the middle plane. From Figure 17 a plane view ( $x-\theta$ plane) is given to examine the acoustic pressure distributions. A complex pattern is formed behind the bifurcations. The maximum SPL values are $119.0 \mathrm{~dB}$ and $113.3 \mathrm{~dB}$ for the bifurcation and the clean duct cases respectively giving a $5.7 \mathrm{~dB}$ SPL increase. The SPL contours shown in Figure 18 confirm that the stronger SPL values appear over the external surface of the core nozzle. For the near field radiation (Figure 19) no doubled $m$ mode structure is observed. The multi- $n$ mode interference is more complicated than the $n=1$ mode case. 


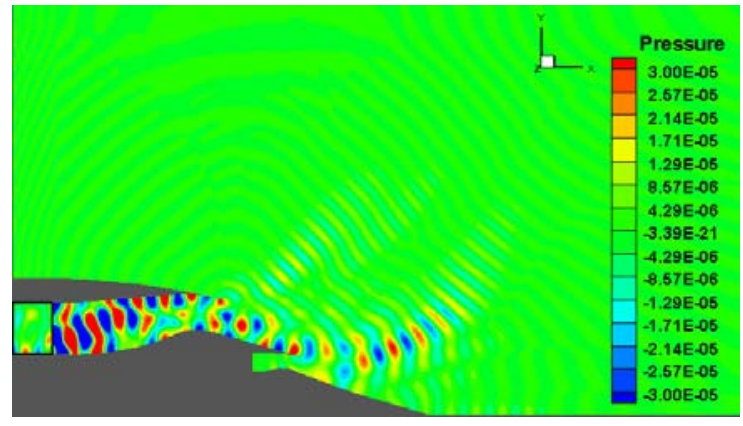

(a) $\theta=0 \operatorname{deg}$

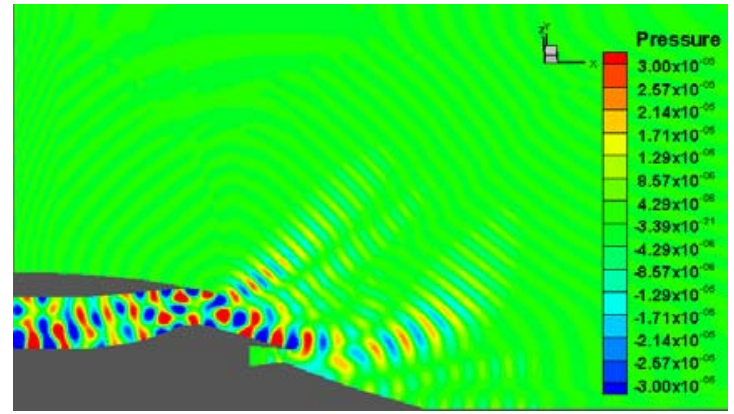

(b) $\theta=45 \mathrm{deg}$

Figure 16 : Acoustic pressure contours.

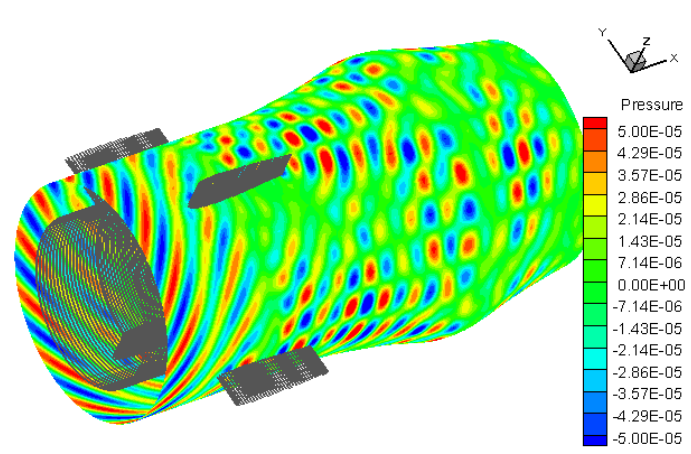

(a) circumferential view

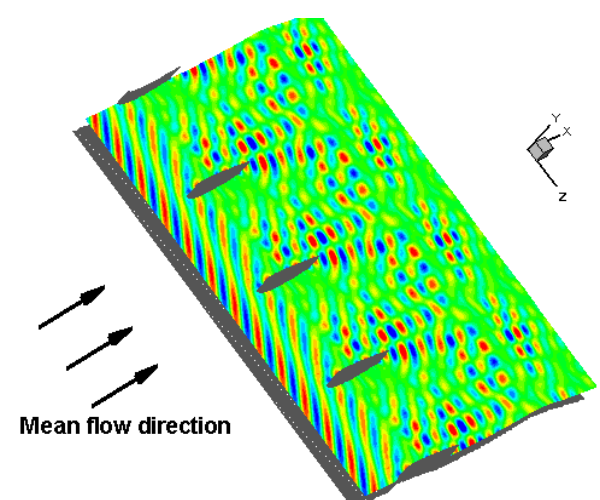

(b) plane ( $x-\theta$ plane) view

Figure 17: A view of acoustic pressures at a cross-section of the bypass duct.

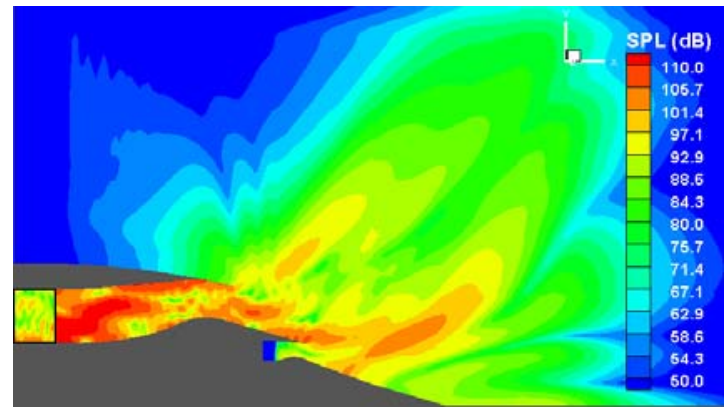

(a) at $\theta=0 \operatorname{deg}$

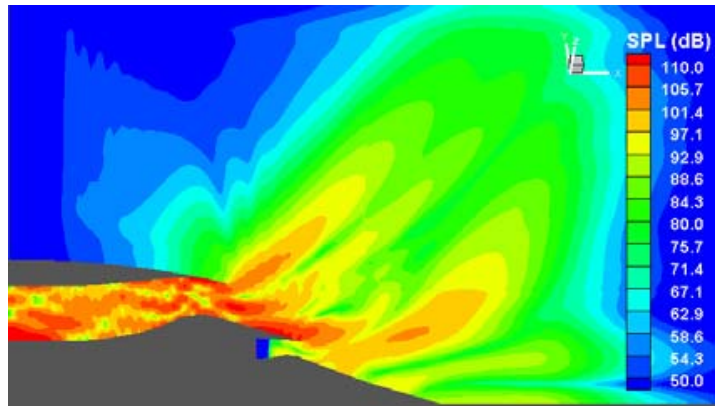

(b) at $\theta=45 \mathrm{deg}$

Figure 18: Near field SPL pattern. 


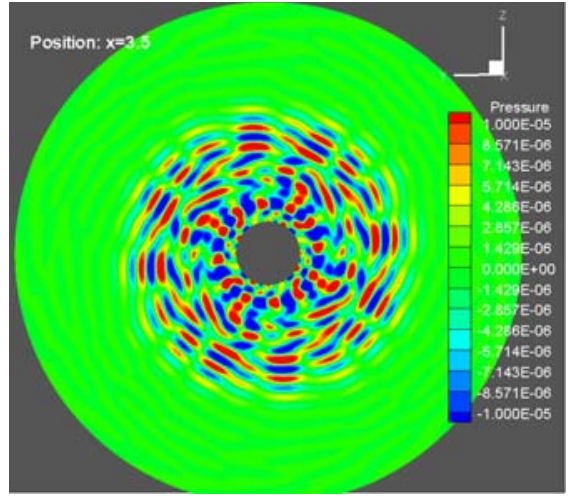

(a) Acoustic pressure

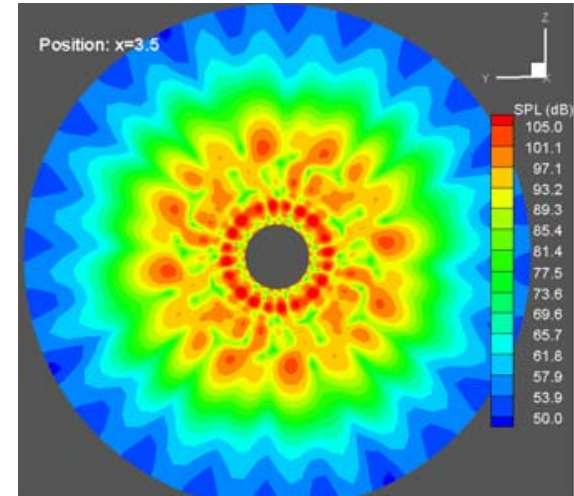

(b) SPL

Figure 19: Acoustic pattern outside the bypass duct at $x / L=3.5$ (position illustrated in Figure 12).

A 3D far field directivity pattern is shown in Figure 20. Again a complex radiation pattern is observed in the far field showing the bifurcation effect. In comparison with the clean duct case, there are additional modes appearing at the lower radiation angles (Figure 21). The bifurcations increased the level of far field sound pressure. The first radiation peak has the maximum value of $68.5 \mathrm{~dB}$ at the $\varphi=39.8 \mathrm{deg}$ which has an increase of $2.0 \mathrm{~dB}$ in comparison with the counterpart in the clean duct case $(66.5 \mathrm{~dB}$ at $\varphi=39.8 \mathrm{deg})$. In comparison with previous works on the bifurcation effect, this work confirms that the bifurcations have influences on the near field sound pressure level through quantitative comparisons with a clean duct case. Furthermore, in this work, the influence of the bifurcations on far field radiation angle and strength are also shown.

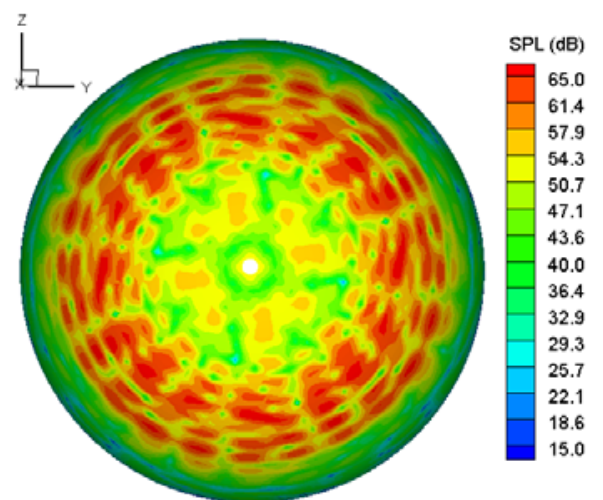

Figure 20: Directivity at far field.

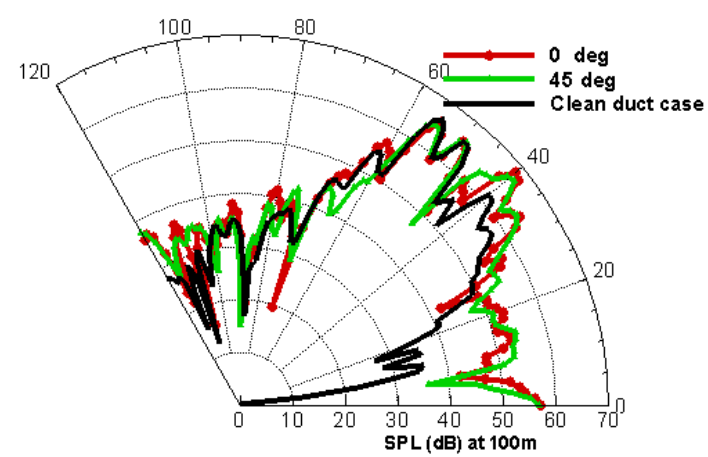

Figure 21: Directivities in the zenith $(\varphi)$ direction. 


\section{Conclusions}

A comparison is firstly made between computations using the APE model and the LEE model in a clean duct case on the 2D computational domain. Results show that the APE simulation leads to a slightly stronger acoustic mode radiation than the LEE result. However the small difference can be neglected. Moreover, there are little difference in the radiation peak angles and interference dip angles by using either model. It appears that both APE and LEE can be used for the bypass duct simulation. The APE model is employed in the subsequent computation for its relatively clear physical meaning.

Acoustic effect of bifurcations in a generic bypass duct is investigated through high-order accurate 3D APE simulations. Four bifurcation arrangements, with an airfoil cross-section, are regularly placed in circumferential direction of the duct. Results of the simulations are compared with those of the clean duct case. In the near field, for the single radial mode $(n=1)$ case, acoustic modes interfere with the bifurcations resulting in a doubled circumferential mode pattern in the SPL distribution. In addition, the bifurcations do not alter the peak radiation angle but increase the sound pressure level. For the multi- $n$ modes case, an additional radiation peak appears at low radiation angle. The sound pressure level is also increased by the bifurcation arrangements. Far field results show the difference between clean duct cases and bifurcation cases more clearly. In the far field, compared to the clean duct case, the main radiation peaks are shifted to a lower radiation angle for both single and multi-n mode cases by the bifurcation arrangements. There are additional modes appearing at the lower observation angles. The levels of the sound pressures at the far field are increased, especially for the multi- $n$ case at low observation angles. In summary, the effect of the bifurcations on the sound radiation on spinning mode sound radiation from the generic engine bypass duct are studied and revealed. 


\section{References}

${ }^{1}$ Baralon, S., Eriksson, L.-E., Billson, M. and Andersson, N., "Evaluation of advanced prediction methods for aero engine exhaust noise," ISABE-2005-1190, 2005.

${ }^{2}$ Zhang, X., Chen, X., Morfey, C., and Nelson, P., "Computation of spinning modal radiation from an unflanged duct," AIAA Journal, 42(9), 2004, pp. 1795-1801.

${ }^{3}$ Huang X., Chen, X., Ma Z.K. and Zhang, X., "Computation of spinning modal radiation through an aircraft engine exhaust," AIAA Journal, 2008, to appear.

${ }^{4}$ Ewert, R. and Schröder, W., "Acoustic perturbation equations based on flow decomposition via source filtering," Journal of Computational Physics, 188, 2003, pp. 365-398.

${ }^{5}$ Nark D. M., Farassat, F., Pope, S. and Vatsa, V., "Effects of bifurcations on aft-fan engine nacelle noise", AIAA Paper 2004-2988, 2004.

${ }^{6}$ Sugimoto R., Astley, R. J., Gabard, G. and Tsuchiya N., "Three-dimensional effects of geometries and acoustic treatments on bypass-duct noise," AIAA Paper 2007-3549, 2007.

${ }^{7} \mathrm{Hu}, \mathrm{F}$., Hussaini, M., and Manthey, J., "Low-dissipation and -dispersion Runge-Kutta schemes for computational acoustics," Journal of Computational Physics, 124, 1996, pp. 177-191.

${ }^{8}$ Hixon, R., "Prefactored Small-Stencil Compact Schemes," Journal of Computational Physics, Vol. 165, No. 2, 2000, pp. 522-541.

${ }^{9}$ Kennedy, C. A. \& Carpenter, M. H., "Comparison of several numerical methods for simulation of compressible shear layers," NASA Technical Paper 3484, 1997.

${ }^{10}$ Farassat, F. and Succi, G.P., "The prediction of helicopter discrete frequency noise," Vertica, 7(4), 1983, pp. 309-320. 